\title{
Asymptomatic recurrence detection and cost-effectiveness in urothelial carcinoma
}

\author{
Hiromichi Iwamura $^{1,2} \cdot$ Shingo Hatakeyama ${ }^{1}\left[\right.$ Makoto Sato $^{2} \cdot$ Chikara Ohyama $^{1}$
}

Received: 13 April 2018 / Accepted: 3 May 2018 / Published online: 9 May 2018

(c) The Author(s) 2018

\begin{abstract}
For the management of muscle-invasive bladder cancer or upper tract urothelial carcinoma, the set guidelines recommend regular surveillance after radical cystectomy or radical nephroureterectomy. However, the prognostic benefit of regular oncological surveillance remains controversial in the absence of prospective studies although several retrospective studies with relatively large sample sizes have demonstrated the association between asymptomatic recurrence and better oncological outcomes. Seven out of eight studies reported that patients diagnosed with symptomatic recurrence showed significantly poorer prognosis in comparison to those diagnosed with asymptomatic recurrence. However, potential lead-time and lengthtime biases prevent the determination of any benefit of regular surveillance. In addition, an optimal surveillance protocol has yet to be established because conventional pathology-based protocols cannot identify the heterogenetic tumor biology of urothelial carcinoma, such as rapid- or slow-growing form of the disease. Several studies suggest that conventional pathology-based surveillance resulted in reduced cost-effectiveness. Recurrence risk-score stratified surveillance protocol including clinical and pathological factors may improve cost-effectiveness. The establishment of optimal risk stratification and surveillance strategies are required to improve the efficacy of regular oncological surveillance. Well-planned prospective studies are necessary to address the prognostic benefit of regular oncological surveillance and shared decision making.
\end{abstract}

Keywords Cystectomy $\cdot$ Nephroureterectomy $\cdot$ Urothelial carcinoma $\cdot$ Recurrence $\cdot$ Surveillance $\cdot$ Symptomatic $\cdot$ Costeffectiveness

\section{Introduction}

Radical cystectomy (RC) and radical nephroureterectomy (RNU) are standard therapy for muscle-invasive bladder cancer (MIBC) and upper tract urothelial carcinoma (UTUC), respectively [1-8]. Because recurrence after radical surgery for urothelial carcinoma leads to poor prognosis, early

Shingo Hatakeyama

shingoh@hirosaki-u.ac.jp

Hiromichi Iwamura

hiro_hiro388@yahoo.co.jp

Makoto Sato

ms.hifu@tohoku-mpu.ac.jp

Chikara Ohyama

coyama@hirosaki-u.ac.jp

1 Department of Urology, Hirosaki University Graduate School of Medicine, 5 Zaifu-cho, Hirosak 036-8562, Japan

2 Department of Urology, Tohoku Medical and Pharmaceutical University, 1-15-1 Fukumuro, Sendai 983-8536, Japan detection followed by salvage therapies is generally considered important [5, 9-12]. Whether regular surveillance after RC for MIBC or RNU for UTUC could improve oncological outcomes remains controversial due to surveillance-related biases and the lack of prospective studies. Additionally, most studies fail to demonstrate any survival benefit of regular surveillance in colorectal cancer [13], breast cancer [14], endometrial cancer [15], or lung cancer [16]. Similarly, debates continue on whether regular oncological surveillance to detect asymptomatic recurrence after RC or RNU improves patient survival $[17,18]$. Furthermore, cost-effectiveness represents another important factor to consider for regular surveillance. Although larger number of screens increase the medical cost, less screening could translate into missing a chance for therapy. Several guidelines recommend regular oncological surveillance $[5,10-12]$; however, these guidelines do not address medical cost. Currently, no established cost-effective surveillance protocol after RC or RNU is available $[5,19,20]$, and only few studies have investigated a cost-effective surveillance protocol after RC [21] and 
RNU [22]. This review summarizes the current evidences of the benefit and cost-effectiveness of regular oncological surveillance to detect recurrence after RC and RNU.

\section{Asymptomatic recurrence and oncological outcomes}

\section{MIBC}

Despite advances in surgical techniques and neoadjuvant/ adjuvant chemotherapies for MIBC, approximately 38-49\% of patients experience disease recurrence within 10 years after RC [2, 9, 23]. Once metastatic disease has occurred, its prognosis is dismal due to limited and transient benefit of salvage therapy, and the median survival after recurrence is approximately 12-14 months [24]. Therefore, the benefit of regular oncological follow-up to detect asymptomatic recurrence has been questioned. To date, seven retrospective studies have investigated the impact of detecting asymptomatic recurrence at regular surveillance after RC [17, 25-30] (Table 1; Fig. 1). Of the seven studies, only one by Volkmer et al. [25] reported no survival benefit of detecting asymptomatic recurrence and concluded that symptom-guided follow-up may provide similar results at lower cost. However, the authors excluded secondary urothelial recurrence from all postoperative recurrences, potentially underestimating the frequency of asymptomatic recurrences and lowering the oncological benefit of regular surveillance. Furthermore, they concluded the lack of benefit of detecting asymptomatic recurrence based on only a log-rank test without multivariate analysis. Conversely, the recent six studies using multivariate Cox regression analysis demonstrated the benefit of detecting asymptomatic recurrence [17, 26-30]. Giannarini et al. [26] reviewed 479 patients who underwent $\mathrm{RC}$ with orthotopic ileal neobladder reconstruction and showed that patients diagnosed with symptomatic recurrence during regular surveillance had significantly worse cancer-specific survival (CSS) and overall survival (OS) compared to those diagnosed with asymptomatic recurrence [CSS: hazard ratio (HR) $1.54 ; P=0.013$ and OS: HR $1.51 ; P=0.015]$ (Fig. 1). Boorjian et al. [27] retrospectively investigated 1599 patients who underwent RC and demonstrated that symptomatic recurrence was an independent risk factor for worse OS (HR 1.59; $P=0.0001$ ) (Fig. 1). Nieuwenhuijzen et al. [28] also reviewed 343 patients treated with $\mathrm{RC}$ and reported that symptomatic recurrence was adversely associated with CSS (HR 2.40; $P=0.013$ ) (Fig. 1). Alimi et al. [29] reported the prognostic disadvantage of symptomatic recurrence in a series of 331 patients who underwent RC (CSS: HR 1.81; $P=0.049$ ) (Fig. 1). Similarly, Osterman et al. [30] reviewed 463 patients who underwent RC and showed worse OS in the symptomatic group than in the asymptomatic group (HR 1.74; $P<0.05$ ) (Fig. 1). Furthermore, Kusaka et al. [17] evaluated the impact of symptomatic recurrence after RC in 581 patients. Their results showed that $53 \%$ of patients presented with symptoms at recurrence after RC and found that patients with symptomatic recurrence had a significantly worse prognosis than with asymptomatic recurrence. Patients with an asymptomatic recurrence frequently

Table 1 Summary of previous studies for prognostic risk of symptomatic recurrence after radical cystectomy or radical nephroureterectomy

\begin{tabular}{|c|c|c|c|c|c|}
\hline Authors (year) & No. of patients & $\begin{array}{l}\text { No. of patients } \\
\text { with recurrence } \\
\text { (\%) }\end{array}$ & $\begin{array}{l}\text { Symptomatic ver- } \\
\text { sus asymptomatic } \\
(\%)\end{array}$ & $\begin{array}{l}\text { Prognostic risk of sympto- } \\
\text { matic recurrence }\end{array}$ & Analysis \\
\hline \multicolumn{6}{|l|}{ Bladder cancer } \\
\hline Volkmer et al. (2009) & 1,270 & $444(49 \%)$ & $65 \%$ versus $35 \%$ & No (not significant in OS) & Univariate, log rank test \\
\hline Giannarini et al. (2010) & 479 & $174(36 \%)$ & $50 \%$ versus $50 \%$ & $\begin{array}{l}\text { Yes (HR 1.51, } P=0.015 \\
\text { in OS) }\end{array}$ & $\begin{array}{l}\text { Multivariate Cox regression } \\
\text { analysis }\end{array}$ \\
\hline Boorjian et al. (2011) & 1,599 & $606(38 \%)$ & $77 \%$ versus $23 \%$ & $\begin{array}{l}\text { Yes (HR 1.59, } P=0.0001 \\
\text { in OS) }\end{array}$ & $\begin{array}{l}\text { Multivariate Cox regression } \\
\text { analysis }\end{array}$ \\
\hline $\begin{array}{l}\text { Nieuwenhuijzen et al. } \\
\text { (2013) }\end{array}$ & 343 & $158(46 \%)$ & $64 \%$ versus $36 \%$ & $\begin{array}{l}\text { Yes (HR 2.40, } P=0.013 \\
\text { in CSS) }\end{array}$ & $\begin{array}{l}\text { Multivariate Cox regression } \\
\text { analysis }\end{array}$ \\
\hline Alimi et al. (2016) & 331 & $160(49 \%)$ & $81 \%$ versus $19 \%$ & $\begin{array}{l}\text { Yes (HR 1.81, } P=0.040 \\
\text { in CSS) }\end{array}$ & $\begin{array}{l}\text { Multivariate Cox regression } \\
\text { analysis }\end{array}$ \\
\hline Kusaka et al. (2017) & 581 & $175(30 \%)$ & $53 \%$ versus $47 \%$ & $\begin{array}{l}\text { Yes (HR 1.94, } \mathrm{P}<0.001 \\
\text { in OS) }\end{array}$ & $\begin{array}{l}\text { IPTW-adjusted multivariate } \\
\text { Cox regression analysis }\end{array}$ \\
\hline Osterman et al. (2017) & 463 & $197(43 \%)$ & $54 \%$ versus $36 \%$ & $\begin{array}{l}\text { Yes (HR } 1.74, \mathrm{P}<0.05 \\
\text { in OS) }\end{array}$ & $\begin{array}{l}\text { Multivariate Cox regression } \\
\text { analysis }\end{array}$ \\
\hline \multicolumn{6}{|l|}{ UTUC } \\
\hline Horiguchi et al. (2017) & 415 & $108(26 \%)$ & $43 \%$ versus $57 \%$ & $\begin{array}{l}\text { Yes (HR 2.08, } P=0.009 \\
\text { in OS) }\end{array}$ & $\begin{array}{l}\text { IPTW-adjusted multivariate } \\
\text { Cox regression analysis }\end{array}$ \\
\hline
\end{tabular}

OS overall survival, CSS cancer-specific survival, $H R$ hazard ratio, IPTW inverse probability of treatment weighting 
Fig. 1 Summary of previous studies aimed at investigating the impact of detecting asymptomatic recurrence during regular surveillance after radical cystectomy. Multivariate Cox regression analysis shows that patients diagnosed with symptomatic recurrence during regular surveillance have significantly worse prognosis compared to those diagnosed with asymptomatic recurrence

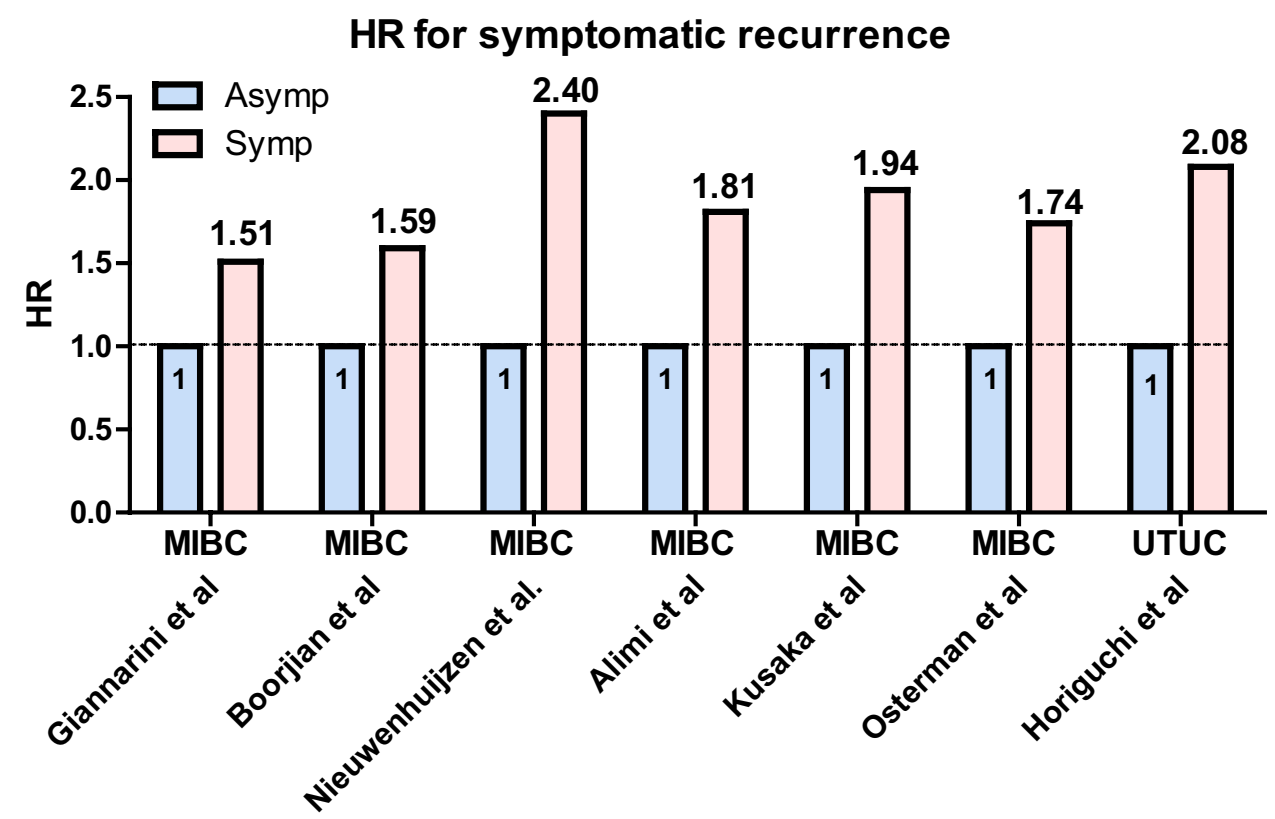

experienced lymph node recurrence, whereas symptomatic patients exhibited larger number of local pelvic recurrence cases. In addition, they used an inverse probability of treatment weighting (IPTW) strategy to remove the effects of confounding factors. The IPTW-adjusted Cox regression analysis performs reweighting of affected and unaffected groups to emulate a propensity score-matched population [31] in order to evaluate the impact of symptomatic recurrence on prognosis. The IPTW-adjusted analysis showed that symptomatic recurrence was an independent risk factor for OS after RC (HR 1.94; $P<0.001)$ and OS after recurrence (HR 2.18; $P<0.001$ ). From these retrospective studies, asymptomatic recurrence detection is suggested as an independent prognostic factor after RC (Fig. 1). Taken together, these results suggest a clinical benefit of regular surveillance after RC; however, prospective studies are needed to confirm its potential benefit.

\section{UTUC}

The standard treatment for non-metastatic UTUC is RNU with bladder cuff excision $[5,7,8,11]$, and regular surveillance after RNU is considered necessary. Although the rationale for regular surveillance is a better prognosis based on earlier recurrence detection, evidence proving the benefit of detecting asymptomatic recurrence after RNU is scarce. To date, only one study has investigated the clinical benefit of regular surveillance after RNU [18] (Table 1; Fig. 1). Horiguchi et al. [18] retrospectively reviewed 415 patients treated with RNU for UTUC at four hospitals in Japan. Their cohort included 108 (26\%) patients with tumor recurrence. The number of patients with asymptomatic and symptomatic recurrences was 62/108 (57\%) and 46/108 (43\%) patients. Their results showed that patients with symptomatic recurrence had a significantly worse overall survival than those with asymptomatic recurrence [18]. These findings are consistent with those of our previous study on symptomatic recurrence after RC [17]. Recurrence-free survival, CSS after RNU, and OS after recurrence were significantly longer in patients in the asymptomatic group than in those in the symptomatic group. IPTW-adjusted multivariate Cox regression analysis showed that symptomatic recurrence was an independent risk factor for OS after RNU (HR 1.75; $P=0.040)$ and OS after recurrence (HR 2.08; $P=0.009$ ) (Fig. 1). Therefore, regular oncological surveillance for detecting asymptomatic recurrence after RNU potentially improves prognosis. Although a prospective randomized study is required, accumulation of retrospective studies is also needed because UTUC is a relatively rare disease [5, 32].

\section{Impact of lead-time bias on outcomes}

On regarding the oncological benefit of regular surveillance for asymptomatic recurrence detection, lead-time bias must be considered. Lead-time bias means that the survival duration after asymptomatic recurrence may be overestimated because surveillance-detected recurrence is generally detected earlier than symptomatic recurrence. Although there are no prospective studies to resolve lead-time bias in regular follow-up after RC or RNU, Osterman et al. [30] retrospectively attempted to account for lead-time bias in patients who underwent RC. They showed that symptomatic 
recurrence was diagnosed 1.7 months before asymptomatic recurrence; nevertheless, median survival after symptomatic recurrence was 8.2 month shorter than after asymptomatic recurrence. These results suggest that detecting asymptomatic recurrence after $\mathrm{RC}$ represents an oncological benefit, which cannot be explained by lead-time bias.

\section{Risk factors for symptomatic recurrence}

Symptomatic recurrence represents a failure of regular surveillance when it occurs through the regular follow-up examinations. Identifying the risk factors for symptomatic recurrence could contribute to improving the surveillance protocol, resulting in better oncological outcomes after RC or RNU. Recently, Anan et al. [6] retrospectively investigated the risk factors for symptomatic recurrence in 581patients after RC, including 100 symptomatic and 75 asymptomatic recurrences. Symptomatic recurrences were significantly frequent within 24 months after RC. Multivariate Cox regression analysis identified lymphovascular invasion (LVI) as an independent risk factor for symptomatic recurrence. In addition, several previous studies [33-39] suggested the association between preoperative severe renal insufficiency and symptomatic recurrence in patients with urothelial carcinoma. Because no study has investigated the relationship between renal insufficiency and symptomatic recurrence, we analyzed the impact of preoperative eGFR status on mode of recurrence in the same cohort reported by Momota et al. (MIBC, $n=610$; UTUC, $n=456$ ) [39]. Our analysis suggests that preoperative eGFR was not significantly different between patients with asymptomatic and symptomatic recurrence (Fig. 2a) although it was significantly lower in patients with recurrence compared to patients without recurrence (Fig. 2b). In addition, multivariate Cox regression analysis showed that eGFR was not an independent factor for symptomatic recurrence in both MIBC and UTUC. These results implied that preoperative eGFR could not identify the high-risk patients with symptomatic recurrence after radical cystectomy or nephroureterectomy. In contrast, the use of neoadjuvant chemotherapy (NAC) and pathological risk associated (pT3-4, LVI, or pN+) were identified as independent factors for symptomatic recurrence in both MIBC and UTUC (Table 2; Fig. 3a, b). These results suggest a significant impact of NAC on symptomatic recurrence in urothelial carcinoma, although previous studies suggested significant risk reduction of NAC use for tumor recurrence in both MIBC [40-42] and UTUC [43-45]. One possible reason is selection of the malignant clone during NAC for urothelial carcinoma. Recent study suggested a potential association between the MIB1 index (immunostaining for Ki67), a proliferation marker, and NAC use [43]. Hosogoe et al. reported the MIB1 index was significantly higher in
Table 2 Multivariate Cox regression analysis for symptomatic recurrence after radical cystectomy $(n=610)$ or radical nephroureterectomy $(n=456)$

\begin{tabular}{lllll}
\hline & Risk factor & $P$ value & HR & $95 \%$ CI \\
\hline MIBC & & & & \\
Age & Continuous & 0.247 & 1.01 & $0.99-1.04$ \\
Sex & Male & 0.193 & 0.73 & $0.45-1.17$ \\
CVD & Positive & 0.880 & 1.04 & $0.62-1.76$ \\
DM & Positive & 0.595 & 1.19 & $0.63-2.26$ \\
Preoperative & Continuous & 0.765 & 1.00 & $0.99-1.01$ \\
$\quad$ eGFR & & & & \\
NAC & Underwent & 0.025 & 1.64 & $1.06-2.53$ \\
Urinary diversion & Ileal neobladder & 0.483 & 0.86 & $0.56-1.31$ \\
Pathological risk & pT3-4, LVI, or & 0.029 & 1.67 & $1.05-2.64$ \\
& pN+ & & & \\
UTUC & & & & \\
Age & Continuous & 0.838 & 1.00 & $0.96-1.03$ \\
Sex & Male & 0.645 & 0.87 & $0.48-1.58$ \\
CVD & Positive & 0.138 & 1.76 & $0.83-3.70$ \\
DM & Positive & 0.734 & 1.18 & $0.45-3.08$ \\
Preoperative & Continuous & 0.857 & 1.00 & $0.97-1.02$ \\
$\quad$ eGFR & & & & \\
NAC & Underwent & 0.031 & 2.08 & $1.07-4.04$ \\
Pathological risk & pT3-4, LVI, or & 0.001 & 1.37 & $1.14-1.65$ \\
& pN+ & & & \\
\hline
\end{tabular}

$C V D$ cardiovascular disease, $D M$ diabetes mellitus, $e G F R$ estimated glomerular filtration rate, $N A C$ neoadjuvant chemotherapy

patients with UTUC with NAC compared to those without NAC [43]. The median MIB1 index was significantly higher in the patients with NAC (21\%; IQR 6.9-44\%) than in those without NAC (3.3\%; IQR 1.9-12\%) (Fig. 4a). In addition, the median MIB1 index was significantly higher in patients with relapse than in those without relapse in the patients with NAC (16\% vs. 39\%) but did not differ in those without NAC (4.0\% vs. $2.4 \%$ ) (Fig. 4b). The authors suggested that MIB1 index $>20 \%$ was significantly associated with poor progression-free survival in patients with UTUC with NAC (Fig. 4c). Therefore, selection of the malignant clone during NAC may impact tumor aggressiveness and resulted in symptomatic recurrence, whereas the total number of recurrence events decreased after NAC [43]. Although further studies are necessary to elucidate the relationship between symptomatic recurrence and NAC use, close attention is recommended when patients with urothelial carcinoma have LVI and higher MIB1 index after RC or RNU.

Although detection of asymptomatic recurrence is associated with improved patient survival [17, 18, 26-30], the underlying reason may be linked to identifying the difference between rapid- and slow-growing tumors (i.e., length-time bias). In fact, the clinical characteristics of symptomatic and asymptomatic recurrence are different. As a previous study 
Fig. 2 Association between renal function and mode of recurrence. Preoperative eGFR is not significantly different between patients with asymptomatic and symptomatic recurrence (a). Preoperative eGFR is significantly lower in patients with recurrence than in those without recurrence (b)

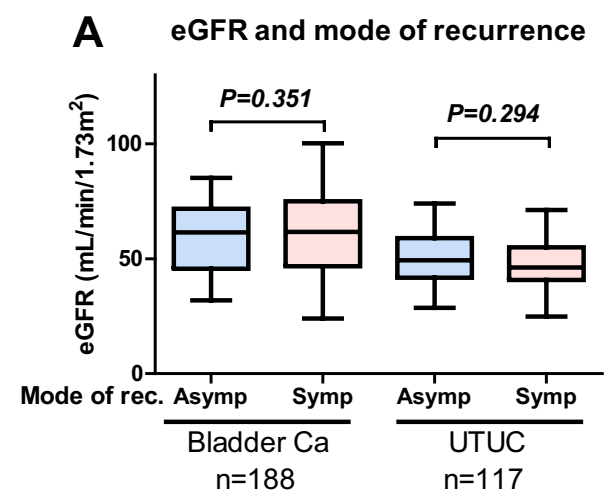

Fig. 3 Potential risk factors for symptomatic recurrence. Multivariate Cox regression analysis shows that neoadjuvant chemotherapy (NAC) and the presence of pathological risk (pT3-4, LVI, or pN+) are independent factors for symptomatic recurrence in $\operatorname{MIBC}(\mathbf{a})$ and UTUC (b)
A Risk for symp. recurrence (MIBC)
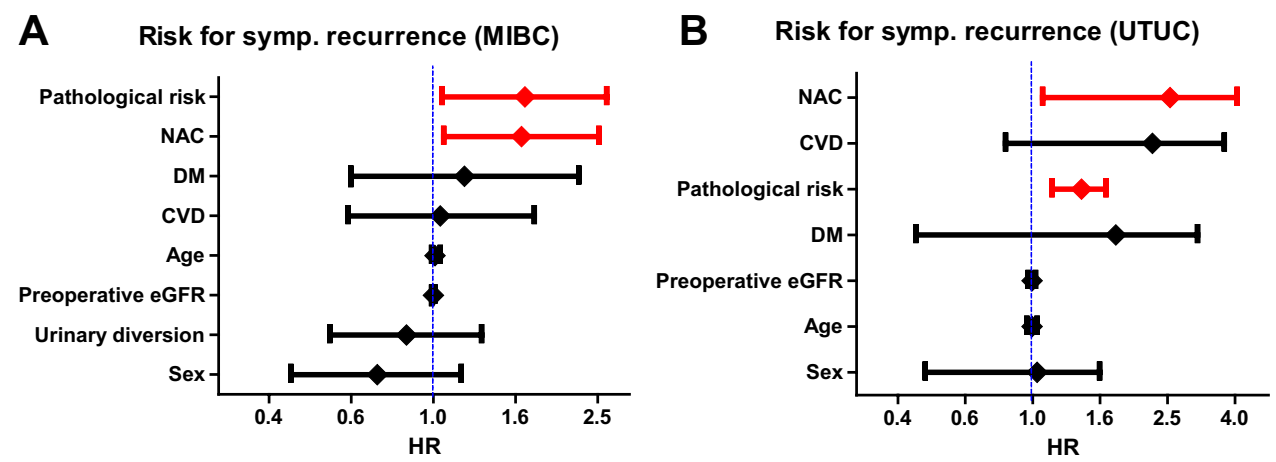

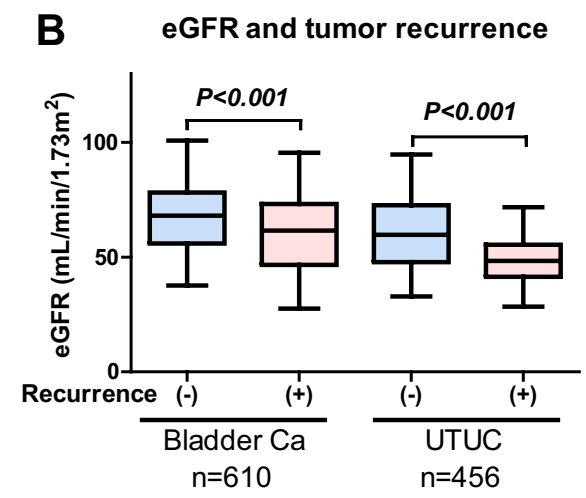

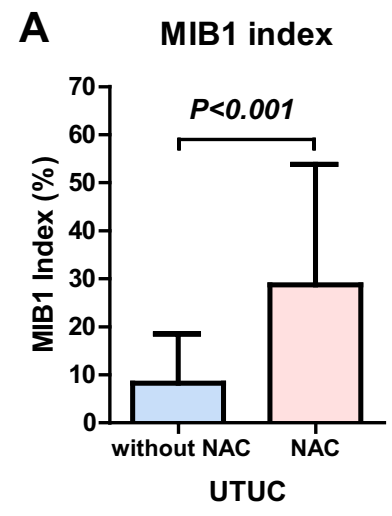

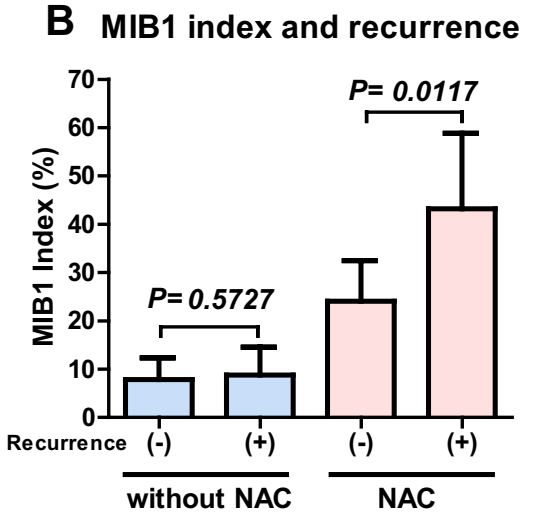

C

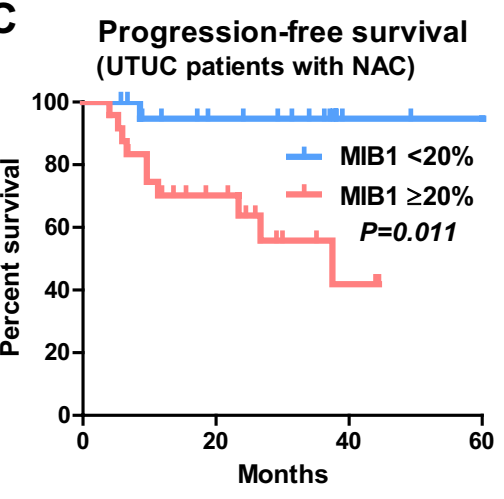

Fig. 4 Association between MIB1 index and neoadjuvant chemotherapy (NAC) in patients with UTUC. MIB1 index is significantly higher in patients with UTUC treated with NAC (21\%; IQR 6.9-44\%) than in those without NAC (3.3\%; IQR 1.9-12\%) (a). The median MIB1 index is significantly higher in patients with NAC with relapse

suggested, symptomatic recurrences are more frequent in local pelvis and/or bone. However, asymptomatic recurrence are more frequently lymph node recurrences [17]. Tumor progression that is missed during routine follow-up suggests a biological heterogeneity between rapid- and slow-growing tumors that cannot be detected by a conventional pathological examination. In addition to clinical or pathological information, novel biomarkers predicting the malignant than in those without relapse (16\% vs. $39 \%$ ) but did not differ in those without NAC (4.0\% vs. $2.4 \%$ ) (b). MIB1 index $>20 \%$ was significantly associated with poor progression-free survival in patients with UTUC treated with NAC (C)

potential of tumors are required for a better understanding of tumor biology. In recent times, the wide-spreading technology of gene testing may allow to predict organs that are likely to metastasize. Genome-based molecular is one of the potential biomarkers for phenotype classification [46-49]. The basal type of MIBC is associated with worse diseasespecific and OS due to its highly invasive and metastatic potential [46]. This phenotype is also associated with an 
epithelial-mesenchymal transition and bladder cancer stemcell biomarkers [50]. Furthermore, the earlier detection of recurrence is pivotal for the possibility to use immunecheckpoint inhibitors $[51,52]$ that potentially provide longterm survival for selected patients. Novel biomarkers predicting the malignant potential of tumors can applied for surveillance schedules in future.

\section{Cost-effectiveness in regular follow-up after surgery}

Expensive follow-up cost is justified only when surveillance leads to improved patient survival. Asymptomatic recurrence identification implies effectiveness of regular surveillance; however, there are no clear guidelines on how to appropriately follow-up patients after RC [20] or RNU [5, 19, 53]. Based on the principle that most recurrences after RC or RNU tend to occur within 2 years [9, 21, 22], several follow-up protocols have been suggested by guidelines [ 5 , 10-12, 53] based on the low level of evidence, and these protocols are mainly stratified by pathological stage without considering the heterogeneity in patients who underwent $\mathrm{RC}$ or RNU. With more screening, asymptomatic recurrence can be detected earlier; however, cost will increase accordingly. A few studies have investigated the cost-effectiveness of surveillance protocols [21, 22, 54]. Vemana et al. [54] calculated the surveillance cost for 24 months after RC using the Surveillance, Epidemiology, and End Results data base. The actual follow-up cost per patient for 24 months was \$1108, and follow-up cost will increase up to 10.6-fold if surveillance were performed based on current established guidelines [9-12, 55]. Kusaka et al. [21] developed a risk-score-stratified surveillance protocol with improved cost-effectiveness after RC. They retrospectively reviewed 581 patients after $\mathrm{RC}$ with regular oncological follow-up. The risk-scores were calculated by summing 6 risk factors $(\mathrm{LVI}+, \mathrm{pN}+, \geq \mathrm{pT} 3$ or surgical margin $[\mathrm{SM}]+$, preoperative chronic kidney disease $[\mathrm{CKD}]+$, cardiovascular disease+, and non-neobladder) that were independently associated with recurrence-free survival by multivariate analysis. Patients were classified into 3 groups; low-risk (0-1), intermediate-risk (2-3), and high-risk (4-6) groups (Table 3) [21]. Based on this risk stratification, the authors developed a risk-score-based protocol for oncological follow-up and evaluated the per-capita cost of recurrence detection. The estimated per-capita cost of recurrence detection was compared between the risk-score-based protocol and the conventional pathology-based protocol. The risk-scorebased protocol led to a dramatic cost reduction compared to the pathology-based protocol. The total estimated 5-year screening cost was 1.9-fold higher with the pathology-based protocol $(\$ 1,148,687)$ than with the risk-score-based protocol $(\$ 613,901)$. Estimated cost differences reached \$534,786 per recurrence detected, a suggested $48 \%$ reduction in this cohort (Fig. 5a) [21].

With respect to UTUC, only one study has investigated the cost-effectiveness of regular oncological surveillance. Momota et al. [22] developed a risk-score-stratified surveillance protocol with improved cost-effectiveness after RNU. They retrospectively reviewed 426 patients after RNU with regular oncological follow-up. Risk-scores were calculated by summing 7 risk factors $(\mathrm{SM}+, \mathrm{LVI}+$, $\geq$ pT3, preoperative $\mathrm{CKD}+, \mathrm{cN}+$ or $\mathrm{pN}+$, hydronephrosis + , and tumor in ureter) that were independently associated with recurrence-free survival by multivariate analysis. Patients were classified into 3 groups; low-risk (0-2), intermediate-risk (3-5), and high-risk (6-12) groups (Table 4) [22]. Based on this risk stratification, they developed a risk-score-based protocol for oncological

Table 3 Risk-score-based classification for MIBC

\begin{tabular}{lll}
\hline Variable & Status & Risk-score \\
\hline Cardiovascular disease & Positive & 1 \\
Preoperative CKD & Positive & 1 \\
Urinary diversion & Non-neobladder & 1 \\
Pathological T stage & $\geq$ pT3 or SM+ & 1 \\
Pathological N stage & pN positive & 1 \\
Lymphovascular invasion & Positive & 1 \\
\hline Risk-score-based classification & & Sum of \\
& & \\
risk- & score \\
\hline Low-risk & & $0-1$ \\
Intermediate-risk & & $2-3$ \\
High-risk & & $4-6$ \\
\hline
\end{tabular}

$C K D$ chronic kidney disease, $S M$ surgical margin 


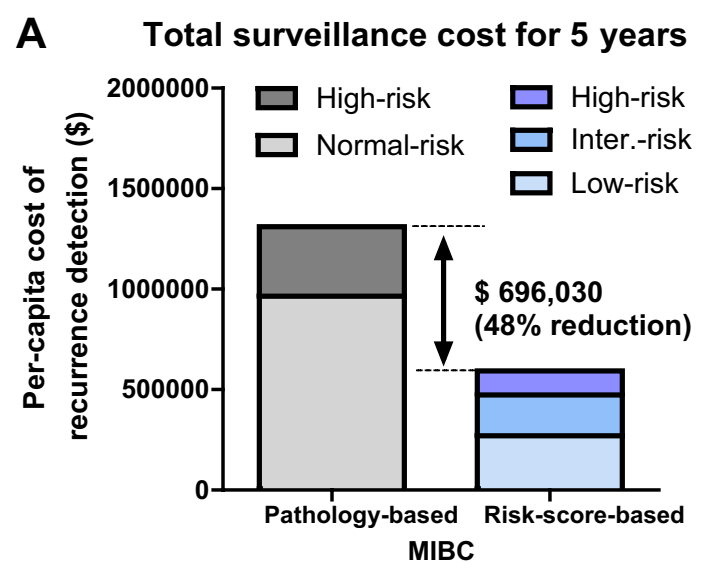

Fig. 5 Comparison of total surveillance cost for 5 years between pathology-based and risk-score-based protocols. The estimated medical cost differences reached $\$ 696,030$ (48\% reduction) in MIBC after

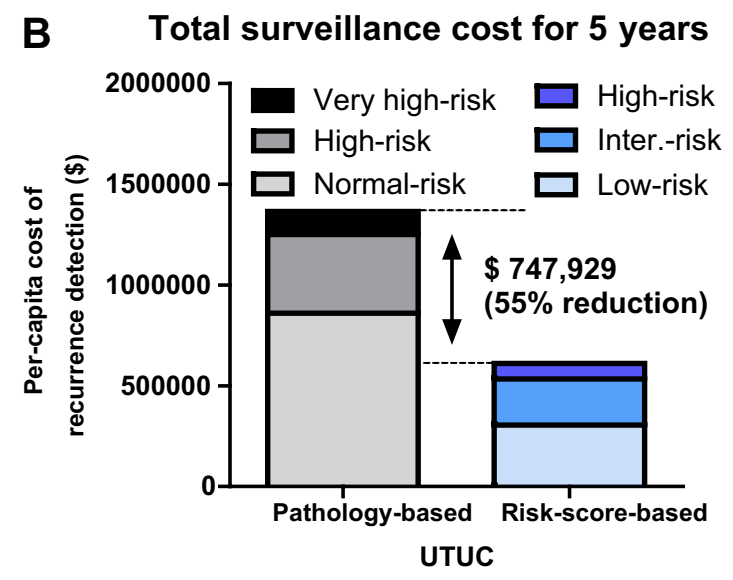

RC (a). The estimated medical cost differences reached $\$ 747,929$ (55\% reduction) in UTUC after RNU (b)

Table 4 Risk-score-based classification for UTUC

\begin{tabular}{llc}
\hline Variable & Status & Risk-score \\
\hline Tumor in ureter & Positive & 1 \\
Hydronephrosis & Positive & 1 \\
Lymph node involvement (cN+ or pN+) & Positive & 2 \\
Preoperative CKD & Positive & 2 \\
Pathological T stage & pT3-4 & 2 \\
Lymphovascular invasion & Positive & 2 \\
Surgical margin & Positive & 2 \\
\hline Risk-score-based classification & & Sum of risk-score \\
\hline Low-risk & & $0-2$ \\
Intermediate-risk & & $3-5$ \\
High-risk & & $6-12$ \\
\hline
\end{tabular}

$C K D$ chronic kidney disease

follow-up and evaluated the per-capita cost of recurrence detection. The estimated per-capita cost of recurrence detection was compared between the risk-score-based protocol and the conventional pathology-based protocol. Their results suggested that the total estimated 5-year surveillance cost was 2.2 -fold higher with the pathologybased protocol $(\$ 1,365,245)$ than with the risk-scorebased protocol $(\$ 617,315)$. Estimated cost differences reached $\$ 747,929$ per recurrence detected, a suggested $55 \%$ reduction in this cohort (Fig. 5b) [22]. These results suggested a higher cost of the conventional pathologybased protocol and the importance of using surveillance protocols considering clinical factors associated with recurrence after RC or RNU.

\section{Limitations}

The present review has several limitations. Firstly, it is an accumulation of retrospective studies with a small number of patients with recurrence. It is difficult to control all variables including selection bias, the influence of leadtime biases, and other unmeasurable confounding factors. Secondly, results cannot be extrapolated independently to other countries because of the difference in health insurance system. Despite these limitations, this review supports the idea that a cost-effective surveillance protocol following curative surgery would detect recurrence at the early stages of disease. In addition, the detection with 
asymptomatic recurrence should secure sufficient time to implement a multimodal therapy after relapse. Although a prospective randomized study comparing a symptombased surveillance would be ideal to clarify the survival benefit of a routine surveillance protocol, a prospective study would be difficult to conduct due to ethical concerns. Therefore, the accumulation of evidences from well-planned retrospective studies is needed to support the clinical benefit of routine surveillance protocol on prognosis and cost-effectiveness.

\section{Conclusion}

Although asymptomatic recurrence detected by regular surveillance potentially results in better oncological outcomes after RC or RNU, the prognostic benefit of regular oncological surveillance remains unclear. The establishment of optimal risk stratification and surveillance strategies are required to improve the efficacy of regular oncological surveillance. Well-planned prospective studies are necessary to address the prognostic benefit of regular oncological surveillance and shared decision making.

Funding This work was supported by a Grant-in-Aid for Scientific Research (Nos. 15H02563, 15K15579, 17K11118, 17K11119, 17K16768, 17K16770, 17K16771, 18K16681, 18K16682, 18K16717, $18 \mathrm{~K} 16718,18 \mathrm{~K} 16719$, and 18K09157) from the Japan Society for the Promotion of Science.

\section{Compliance with ethical standards}

Conflict of interest The authors declare that they have no conflict of interest.

Open Access This article is distributed under the terms of the Creative Commons Attribution 4.0 International License (http://creativeco mmons.org/licenses/by/4.0/), which permits unrestricted use, distribution, and reproduction in any medium, provided you give appropriate credit to the original author(s) and the source, provide a link to the Creative Commons license, and indicate if changes were made.

\section{References}

1. Koie T, Hatakeyama S, Yoneyama T, Ishimura H, Yamato T, Ohyama C. Experience and functional outcome of modified ileal neobladder in 95 patients. Int J Urol. 2006;13(9):1175-9. https:// doi.org/10.1111/j.1442-2042.2006.01525.x.

2. Studer UE, Burkhard FC, Schumacher M, Kessler TM, Thoeny $\mathrm{H}$, Fleischmann A, et al. Twenty years experience with an ileal orthotopic low pressure bladder substitute-lessons to be learned. J Urol. 2006;176(1):161-6. https://doi.org/10.1016/s0022 $-5347(06) 00573-8$.

3. Koie T, Hatakeyama S, Yoneyama T, Hashimoto Y, Kamimura N, Ohyama C. Uterus-, fallopian tube-, ovary-, and vagina-sparing cystectomy followed by U-shaped ileal neobladder construction for female bladder cancer patients: oncological and functional outcomes. Urology. 2010;75(6):1499-503. https://doi.org/10.1016/j. urology.2009.08.083.

4. Koie T, Ohyama C, Yamamoto H, Hatakeyama S, Kudoh S, Yoneyama T, et al. Minimum incision endoscopic radical cystectomy in patients with malignant tumors of the urinary bladder: clinical and oncological outcomes at a single institution. Eur J Surg Oncol. 2012;38(11):1101-5. https://doi.org/10.1016/j. ejso.2012.07.115.

5. Oya M, Kikuchi E. Evidenced-based clinical practice guideline for upper tract urothelial carcinoma (summary-Japanese Urological Association, 2014 edition). Int J Urol. 2015;22(1):3-13. https:// doi.org/10.1111/iju.12630.

6. Anan G, Hatakeyama S, Fujita N, Iwamura H, Tanaka T, Yamamoto $\mathrm{H}$, et al. Risk factors for symptomatic recurrence after radical cystectomy in patients with locally advanced bladder cancer. Eur Urol Suppl. 2018;17(2):e145-e146. https://doi.org/10.1016/ S1569-9056(18)30950-3.

7. Horiguchi H, Yoneyama T, Hatakeyama S, Tokui N, Sato T, Fujita $\mathrm{N}$, et al. Impact of bacillus Calmette-Guerin therapy of upper urinary tract carcinoma in situ: comparison of oncological outcomes with radical nephroureterectomy. Med Oncol. 2018;35(4):41. https://doi.org/10.1007/s12032-018-1102-y.

8. Kido K, Hatakeyama S, Fujita N, Yamamoto H, Tobisawa Y, Yoneyama T, et al. Oncologic outcomes for open and laparoscopic radical nephroureterectomy in patients with upper tract urothelial carcinoma. Int J Clin Oncol. 2018. https://doi.org/10.1007/s1014 7-018-1248-9.

9. Yafi FA, Aprikian AG, Fradet Y, Chin JL, Izawa J, Rendon R, et al. Surveillance guidelines based on recurrence patterns after radical cystectomy for bladder cancer: the Canadian Bladder Cancer Network experience. BJU Int. 2012;110(9):1317-23. https:// doi.org/10.1111/j.1464-410X.2012.11133.x.

10. Kubota Y, Nakaigawa N. Essential content of evidence-based clinical practice guidelines for bladder cancer: The Japanese Urological Association 2015 update. Int J Urol. 2016;23(8):640-5. https ://doi.org/10.1111/iju.13141.

11. Alfred Witjes J, Lebret T, Comperat EM, Cowan NC, De Santis M, Bruins HM, et al. Updated 2016 EAU guidelines on muscleinvasive and metastatic bladder cancer. Eur Urol. 2017;71(3):462 75. https://doi.org/10.1016/j.eururo.2016.06.020.

12. Spiess PE, Agarwal N, Bangs R, Boorjian SA, Buyyounouski MK, Clark PE, et al. Bladder cancer, version 5.2017, NCCN clinical practice guidelines in oncology. J Natl Compr Canc Netw. 2017;15(10):1240-67. https://doi.org/10.6004/jnccn.2017.0156.

13. Jeffery M, Hickey BE, Hider PN, See AM. Follow-up strategies for patients treated for non-metastatic colorectal cancer. Cochrane Database Syst Rev. 2016;11:CD002200. https://doi. org/10.1002/14651858.CD002200.pub3.

14. Moschetti I, Cinquini M, Lambertini M, Levaggi A, Liberati A. Follow-up strategies for women treated for early breast cancer. Cochrane Database Syst Rev. 2016;5:CD001768. https://doi. org/10.1002/14651858.CD001768.pub3.

15. Fung-Kee-Fung M, Dodge J, Elit L, Lukka H, Chambers A, Oliver T. Follow-up after primary therapy for endometrial cancer: a systematic review. Gynecol Oncol. 2006;101(3):520-9. https:// doi.org/10.1016/j.ygyno.2006.02.011

16. Younes RN, Gross JL, Deheinzelin D. Follow-up in lung cancer: how often and for what purpose? Chest. 1999;115(6):1494-9.

17. Kusaka A, Hatakeyama S, Hosogoe S, Hamano I, Iwamura H, Fujita N, et al. Detecting asymptomatic recurrence after radical cystectomy contributes to better prognosis in patients with muscle-invasive bladder cancer. Med Oncol. 2017;34(5):90. https:// doi.org/10.1007/s12032-017-0955-9. 
18. Horiguchi H, Hatakeyama S, Anan G, Kubota Y, Kodama H, Momota M, et al. Detecting asymptomatic recurrence after radical nephroureterectomy contributes to better prognosis in patients with upper urinary tract urothelial carcinoma. Oncotarget. 2018;9(9):8746-55. https://doi.org/10.18632/oncotarget.23982.

19. Seisen T, Colin P, Hupertan V, Yates DR, Xylinas E, Nison L, et al. Postoperative nomogram to predict cancer-specific survival after radical nephroureterectomy in patients with localised and/ or locally advanced upper tract urothelial carcinoma without metastasis. BJU Int. 2014;114(5):733-40. https://doi.org/10.1111/ bju. 12631.

20. Stewart-Merrill SB, Boorjian SA, Thompson RH, Psutka SP, Cheville JC, Thapa P, et al. Evaluation of current surveillance guidelines following radical cystectomy and proposal of a novel risk-based approach. Urol Oncol. 2015;33(8):339e1-8. https://doi. org/10.1016/j.urolonc.2015.04.017.

21. Kusaka A, Hatakeyama S, Hosogoe S, Hamano I, Iwamura H, Fujita N, et al. Risk-stratified surveillance and cost effectiveness of follow-up after radical cystectomy in patients with muscleinvasive bladder cancer. Oncotarget. 2017;8(39):65492-505. https ://doi.org/10.18632/oncotarget.19043.

22. Momota M, Hatakeyama S, Yamamoto H, Iwamura H, Tobisawa Y, Yoneyama T, et al. Risk-stratified surveillance protocol improves cost-effectiveness after radical nephroureterectomy in patients with upper tract urothelial carcinoma. Oncotarget. 2018;9:23047-57. https://doi.org/10.18632/oncotarget.25198.

23. Hautmann RE, Abol-Enein H, Hafez K, Haro I, Mansson W, Mills RD, et al. Urinary diversion. Urology. 2007;69(1 Suppl.):17-49. https://doi.org/10.1016/j.urology.2006.05.058.

24. Sternberg CN, de Mulder PH, Schornagel JH, Theodore C, Fossa SD, van Oosterom AT, et al. Randomized phase III trial of high-dose-intensity methotrexate, vinblastine, doxorubicin, and cisplatin (MVAC) chemotherapy and recombinant human granulocyte colony-stimulating factor versus classic MVAC in advanced urothelial tract tumors: European Organization for Research and Treatment of Cancer Protocol no. 30924. J Clin Oncol. 2001;19(10):2638-46. https://doi.org/10.1200/ jco.2001.19.10.2638.

25. Volkmer BG, Schnoeller T, Kuefer R, Gust K, Finter F, Hautmann RE. Upper urinary tract recurrence after radical cystectomy for bladder cancer-who is at risk? J Urol. 2009;182(6):2632-7. https ://doi.org/10.1016/j.juro.2009.08.046.

26. Giannarini G, Kessler TM, Thoeny HC, Nguyen DP, Meissner C, Studer UE. Do patients benefit from routine follow-up to detect recurrences after radical cystectomy and ileal orthotopic bladder substitution? Eur Urol. 2010;58(4):486-94. https://doi. org/10.1016/j.eururo.2010.05.041.

27. Boorjian SA, Tollefson MK, Cheville JC, Costello BA, Thapa $\mathrm{P}$, Frank I. Detection of asymptomatic recurrence during routine oncological followup after radical cystectomy is associated with improved patient survival. J Urol. 2011;186(5):1796-802. https ://doi.org/10.1016/j.juro.2011.07.005.

28. Nieuwenhuijzen JA, de Vries RR, van Tinteren H, Bex A, Van der Poel HG, Meinhardt W, et al. Follow-up after cystectomy: regularly scheduled, risk adjusted, or symptom guided? Patterns of recurrence, relapse presentation, and survival after cystectomy. Eur J Surg Oncol. 2014;40(12):1677-85. https://doi.org/10.1016/j. ejso.2013.12.017.

29. Alimi Q, Verhoest G, Kammerer-Jacquet SF, Mathieu R, RiouxLeclercq N, Manunta A, et al. Role of routine computed tomography scan in the oncological follow up of patients treated by radical cystectomy for bladder cancer. Int J Urol. 2016;23(10):840-6. https://doi.org/10.1111/iju.13164.

30. Osterman CK, Alanzi J, Lewis JD, Kaufman EL, Narayan $\mathrm{V}$, Boursi B, et al. Association between symptomatic versus asymptomatic recurrence and survival in bladder cancer. Clin Genitourin Cancer. 2017. https://doi.org/10.1016/j. clgc.2017.11.001.

31. Austin PC, Stuart EA. Moving towards best practice when using inverse probability of treatment weighting (IPTW) using the propensity score to estimate causal treatment effects in observational studies. Stat Med. 2015;34(28):3661-79. https://doi.org/10.1002/ sim.6607.

32. Roupret M, Babjuk M, Comperat E, Zigeuner R, Sylvester RJ, Burger M, et al. European association of urology guidelines on upper urinary tract urothelial cell carcinoma: 2015 update. Eur Urol. 2015;68(5):868-79. https://doi.org/10.1016/j.eurur o.2015.06.044.

33. Cao J, Zhao X, Zhong Z, Zhang L, Zhu X, Xu R. Prognostic value of pre-operative renal insufficiency in urothelial carcinoma: a systematic review and meta-analysis. Sci Rep. 2016;6:35214. https:// doi.org/10.1038/srep35214.

34. Li CE, Chien CS, Chuang YC, Chang YI, Tang HP, Kang CH. Chronic kidney disease as an important risk factor for tumor recurrences, progression and overall survival in primary non-muscle-invasive bladder cancer. Int Urol Nephrol. 2016;48(6):993-9. https://doi.org/10.1007/s11255-016-1264-5.

35. Hamano I, Hatakeyama S, Iwamurau H, Fujita N, Fukushi K, Narita T, et al. Preoperative chronic kidney disease predicts poor oncological outcomes after radical cystectomy in patients with muscle-invasive bladder cancer. Oncotarget. 2017;8(37):6140414. https://doi.org/10.18632/oncotarget.18248.

36. Kodama H, Hatakeyama S, Fujita N, Iwamura H, Anan G, Fukushi K, et al. Preoperative chronic kidney disease predicts poor oncological outcomes after radical nephroureterectomy in patients with upper urinary tract urothelial carcinoma. Oncotarget. 2017;8(47):83183-94. https://doi.org/10.18632/oncotarget.20554

37. Yu HS, Hwang JE, Chung HS, Cho YH, Kim MS, Hwang EC, et al. Is preoperative chronic kidney disease status associated with oncologic outcomes in upper urinary tract urothelial carcinoma? A multicenter propensity score-matched analysis. Oncotarget. 2017. https://doi.org/10.18632/oncotarget.16239.

38. Matsumoto A, Nakagawa T, Kanatani A, Ikeda M, Kawai T, Miyakawa J, et al. Preoperative chronic kidney disease is predictive of oncological outcome of radical cystectomy for bladder cancer. World J Urol. 2018;36(2):249-56. https://doi.org/10.1007/ s00345-017-2141-2.

39. Momota M, Hatakeyama S, Tokui N, Sato T, Yamamoto H, Tobisawa $Y$, et al. The impact of preoperative severe renal insufficiency on poor postsurgical oncological prognosis in patients with urothelial carcinoma. Eur Urol Focus. 2018. https://doi. org/10.1016/j.euf.2018.03.003.

40. Koie T, Ohyama C, Yamamoto H, Imai A, Hatakeyama S, Yoneyama T, et al. Neoadjuvant gemcitabine and carboplatin followed by immediate cystectomy may be associated with a survival benefit in patients with clinical T2 bladder cancer. Med Oncol. 2014;31(5):949. https://doi.org/10.1007/s12032-014-0949-9.

41. Ohyama C, Hatakeyama S, Yoneyama T, Koie T. Neoadjuvant chemotherapy with gemcitabine plus carboplatin followed by immediate radical cystectomy for muscle-invasive bladder cancer. Int J Urol. 2014;21(1):3-4. https://doi.org/10.1111/iju.12230.

42. Anan G, Hatakeyama S, Fujita N, Iwamura H, Tanaka T, Yamamoto $\mathrm{H}$, et al. Trends in neoadjuvant chemotherapy use and oncological outcomes for muscle-invasive bladder cancer in Japan: a multicenter study. Oncotarget. 2017;8(49):86130-142. https://doi. org/10.18632/oncotarget.20991.

43. Hosogoe S, Hatakeyama S, Kusaka A, Hamano I, Iwamura $\mathrm{H}$, Fujita N, et al. Platinum-based neoadjuvant chemotherapy improves oncological outcomes in patients with locally advanced 
upper tract urothelial carcinoma. Eur Urol Focus. 2017:231-40. https://doi.org/10.1016/j.euf.2017.03.013.

44. Kubota Y, Hatakeyama S, Tanaka T, Fujita N, Iwamura H, Mikami $\mathrm{J}$, et al. Oncological outcomes of neoadjuvant chemotherapy in patients with locally advanced upper tract urothelial carcinoma: a multicenter study. Oncotarget. 2017;8(60):101500-8. https://doi. org/10.18632/oncotarget.21551.

45. Kobayashi K, Saito T, Kitamura Y, Bilim V, Toba T, Kawasaki T, et al. Effect of preoperative chemotherapy on survival of patients with upper urinary tract urothelial carcinoma clinically involving regional lymph nodes. Int J Urol. 2016;23(2):153-8. https://doi. org/10.1111/iju.13010.

46. Choi W, Porten S, Kim S, Willis D, Plimack ER, Hoffman-Censits J, et al. Identification of distinct basal and luminal subtypes of muscle-invasive bladder cancer with different sensitivities to frontline chemotherapy. Cancer Cell. 2014;25(2):152-65. https:// doi.org/10.1016/j.ccr.2014.01.009.

47. McConkey DJ, Choi W, Dinney CP. Genetic subtypes of invasive bladder cancer. Curr Opin Urol. 2015;25(5):449-58. https://doi. org/10.1097/mou.0000000000000200.

48. Robertson AG, Kim J, Al-Ahmadie H, Bellmunt J, Guo G, Cherniack $\mathrm{AD}$, et al. Comprehensive molecular characterization of muscle-invasive bladder cancer. Cell. 2017;171(3):540e25-556e25. https://doi.org/10.1016/j.cell.2017.09.007.

49. Audenet F, Attalla K, Sfakianos JP. The evolution of bladder cancer genomics: What have we learned and how can we use it? Urol Oncol. 2018. https://doi.org/10.1016/j.urolonc.2018.02.017.
50. Chan KS, Espinosa I, Chao M, Wong D, Ailles L, Diehn M, et al. Identification, molecular characterization, clinical prognosis, and therapeutic targeting of human bladder tumor-initiating cells. Proc Natl Acad Sci USA. 2009;106(33):14016-21. https://doi. org/10.1073/pnas.0906549106.

51. Bellmunt J, de Wit R, Vaughn DJ, Fradet Y, Lee JL, Fong L, et al. Pembrolizumab as second-line therapy for advanced urothelial carcinoma. N Engl J Med. 2017;376(11):1015-26. https://doi. org/10.1056/NEJMoa1613683.

52. Bellmunt J, Powles T, Vogelzang NJ. A review on the evolution of PD-1/PD-L1 immunotherapy for bladder cancer: the future is now. Cancer Treat Rev. 2017;54:58-67. https://doi.org/10.1016/j. ctrv.2017.01.007.

53. Roupret M, Babjuk M, Comperat E, Zigeuner R, Sylvester RJ, Burger M, et al. European association of urology guidelines on upper urinary tract urothelial carcinoma: 2017 update. Eur Urol. 2018;73(1):111-22. https://doi.org/10.1016/j.eururo.2017.07.036.

54. Vemana G, Vetter J, Chen L, Sandhu G, Strope SA. Sources of variation in follow-up expenditure after radical cystectomy. Urol Oncol. 2015;33(6):267 e31-e37. https://doi.org/10.1016/j.urolo nc.2015.03.009.

55. Lerner SP. Bladder cancer: ASCO endorses EAU muscle-invasive bladder cancer guidelines. Nat Rev Urol. 2016;13(8):440-1. https ://doi.org/10.1038/nrurol.2016.114. 